Genre et filiation : pratiques et représentations

\title{
Pedro Almodóvar ou la maternité performée
}

Pedro Almodóvar: maternity as performativity

\section{Belén HERNANDEZ MARZAL}

\section{(2) OpenEdition}

Journals

Édition électronique

URL : http://journals.openedition.org/transtexts/485

DOI : $10.4000 /$ transtexts.485

ISSN : 2105-2549

Éditeur

Gregory B. Lee

Référence électronique

Belén HERNANDEZ MARZAL, «Pedro Almodóvar ou la maternité performée », Transtext(e)s

Transcultures 跨文本跨文化 [En ligne], 8 | 2013, mis en ligne le 02 décembre 2013, consulté le 23 avril 2019. URL : http://journals.openedition.org/transtexts/485; DOI : 10.4000/transtexts.485

\section{(c) Tous droits réservés}




\section{Transtext(e)s Transcultures 跨文本跨 文化}

Journal of Global Cultural Studies

$8 \mid 2013$ :

Genre et filiation : pratiques et représentations

Récits de filiation et (ré)écriture de soi

\section{Pedro Almodóvar ou la maternité performée}

Pedro Almodóvar: maternity as performativity

\section{BELÉN HERNANDEZ MARZAL}

\section{Résumés}

\section{Français English}

La question de la filiation traverse le cinéma de Pedro Almodóvar, que cela soit dans la quête de la mère ou du père (Tout sur ma mère) ou à travers des portraits de bonnes ou mauvaises mères. Devant la crise des modèles traditionnels de couple et de famille, d'autres formes s'imposent comme des alternatives plus viables. Le cinéma du réalisateur espagnol opère ainsi une remise en question de la notion de maternité tout en élargissant les concepts de filiation et de maternité : il nous donne à voir d'autres modèles, de nouvelles figures de la maternité à travers des transsexuels qui deviennent des mères. Sa dédicace à la fin de Tout sur ma mère aux femmes actrices, aux hommes qui jouent à être des femmes prend donc tout son sens car, pour Almodóvar, on ne naît pas $\mathrm{m} /$ père, on le devient.

Kinship is a prevalent theme in the films of Pedro Almodóvar: it can be related to the quest for the mother or the father (All About My Mother) or it appears through portraits of good or bad mothers. With the crisis of traditional models (couple and family), other forms are emerging as more viable alternatives. The films of the Spanish director question motherhood, while it expands concepts of kinship and maternity: it allows us to see other models, new representations of motherhood through transsexuals who become mothers. Therefore his dedicate, at the end of All About My Mother, to women actresses, to men who pretend to be women, makes sense because, for Almodóvar, you were not born a mother / father, you become one. 


\section{Texte intégral}

La maternité (tout comme la filiation) ne semble pas aller de soi dans le cinéma d'Almodóvar. Dans Qu'est-ce que j’ai fait pour mériter ça ?!, Gloria, femme de ménage et épouse frustrée et trompée par son mari, assomme son mari infidèle avec un os de jambon. Elle n'hésite pas à « vendre » l'un de ses enfants à son dentiste pour se payer un moldeador, fer lisseur-onduleur pour les cheveux. Becky, dans Talons aiguilles, abandonne sa fille pour s'enfuir avec son amant... Et pourtant, maternité et filiation sont au cœur de la cinématographie du réalisateur espagnol. ${ }^{1}$ Dans Volver, Raimunda traîne un lourd secret. Violée par son père, elle protège sa fille lorsque celle-ci tue celui qu'elle croyait être son père (en réalité son beau-père), pour esquiver ses avances. Raimunda, mère exemplaire, qualifiée de mère courage par la critique, sera prête à tout pour sa fille, et ira jusqu'à se débarrasser du cadavre de son mari pour la libérer de toute culpabilité. Enfin, Pepa, dans Femmes au bord d'une crise de nerfs, devant l'inconsistance de son amant Iván -qui s'enfuit avec une autre femme- finira par assumer la maternité toute seule.

Ces quelques exemples parmi tant d'autres dans sa filmographie nous amènent à un premier constat : le modèle traditionnel de famille apparaît malmené chez Almodóvar, il se révèle défaillant et mène souvent à l'échec. Le cinéma d'Almodóvar nous est donné à lire comme une quête d'autres possibilités.

3 Le premier film d'Almodóvar où la maternité n'est pas exclusivement le fait de la femme est La loi du désir, où l'on trouve le premier exemple de la maternité comme choix de vie. Dans ce film, la transsexuelle Tina (en réalité Tino) forme un couple lesbien avec la mère d'Ada, une fillette de dix ans qu'elle prend en charge lors des fréquentes absences maternelles, remplaçant avantageusement sa mère biologique. ${ }^{2}$

4 Très féminine, outrageusement maquillée, engoncée dans des robes ultramoulantes, la transsexuelle Tina transcende la féminité, mettant en évidence les mécanismes performatifs du genre. ${ }^{3}$

5 Dans Talons aiguilles, par exemple, Rebeca passe toute sa vie à imiter sa mère Becky. Genre et imitation iraient ainsi de pair : elle copie le style vestimentaire de sa mère, ses bijoux, épouse l'ancien amant de celle-ci et couchera avec Letal, le travesti qu'imite Becky.

$6 \quad$ Ces mécanismes de performativité du genre sont encore plus flagrants chez les transgenres. ${ }^{4}$ Car s'il y a quelque chose qui caractériserait la féminité d'un transsexuel, d'après Almodóvar, ce serait l'artifice, le jeu, comme il le déclare à propos du personnage de Tina. ${ }^{5}$ Et si Almodóvar choisit une femme -au lieu d'un transsexuel- pour jouer Tina, c'est parce que le transsexuel et la femme biologique n'expriment pas la féminité de la même manière, la féminité d'une femme étant, selon lui, beaucoup plus " tranquille et sereine ». ${ }^{6}$ En revanche, ce qu'il voulait pour son film, c'était une femme qu' " incarne la féminité exacerbée, crispée et exhibitionniste d'un transsexuel ».7

$7 \quad$ C'est pour cela qu'il demande à Carmen Maura d'imiter un homme qui imite à son tour une femme dans une série de reflets où on ne sait plus où se situe le vrai, et qui démontre à son tour que le genre est une performance, un ensemble d'actes répétés et qu'il dépend en grande partie de notre perception. ${ }^{8}$ D'ailleurs, lorsque le mari de Rebeca, l'ancien amant de Becky lui demande si « Letal » est masculin ou féminin, il lui dit « ça dépend. Pour toi je suis un 
homme».

8 Le transsexuel, le transgenre chez Almodóvar, est hypertélique, car il grossit « les traits supposés de la féminité, des érotiques aux psychologiques » ${ }^{9}$ Tina est donc consciente de son imitation et de son artificialité. On constate également que, de même que le transsexuel performe le genre, la maternité peut être également performée. Tina, en tant que transsexuelle, performe la féminité, elle en regorge même. Tout comme elle regorge d'amour maternel pour la petite Ada, pour qui elle sera une véritable mère. Comme le rappelle le synopsis du film, elle incarne à la perfection le rôle de mère, elle endosse donc un rôle, elle joue ou performe la maternité. ${ }^{10}$ Elles prieront ensemble, elle s'efforcera de lui donner une éducation et s'inquiétera de ses résultats scolaires - « Tu ne sais pas les notes qu'elle me ramène », se plaint-elle à son frère cinéaste-, comme n'importe quelle mère de famille ou mère au foyer. Elle devient une bonne mère, névrosée, certes, mais aimante, offrant une cellule familiale rassurante mais assez atypique, dans laquelle le rôle du père est tenu par le frère de Tina, homosexuel, dont la petite Ada tombe amoureuse, faisant ainsi son Edipe. L'ordre symbolique est ainsi maintenu, du moins en apparence.

$9 \quad$ Mais là où la famille traditionnelle apparaît complètement chamboulée c'est dans Tout sur ma mère. Résumons un peu l'argument : Manuela, infirmière responsable du don d'organes dans un hôpital de Madrid, vit avec son fils Esteban, apprenti écrivain, qu'elle élève seule et qui n'a jamais connu son père. Désireux de connaître son histoire, il obtient de Manuela la promesse de lui en parler le lendemain. Ce sera, hélas, trop tard car Esteban meurt renversé par une voiture. Manuela part ensuite à Barcelone, à la recherche d'Esteban/Lola, père transsexuel d'Esteban, afin d'exaucer à titre posthume le souhait de son fils. Dans sa quête, elle retrouvera Agrado, amie de longue date, transsexuelle à moitié opérée également, et fera la connaissance de sœur Rosa, enceinte elle aussi de Lola et contaminée par celle-ci du sida. Se sentant une étrangère dans sa propre famille, défaillante et peu aimante à son égard, Rosa demande de l'aide à Manuela. Celle-ci a beau lui répéter qu'elle n'est pas sa mère, rien n'y fait : sœur Rosa a jeté son dévolu sur elle. Finalement Manuela, femme complètement dévouée aux autres, assume cette responsabilité et devient une sorte de mère-sœur-infirmière-épouse pour Rosa. ${ }^{11}$ Elle sera aussi, à la mort de

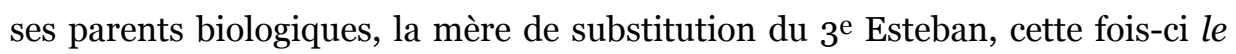
bon, le définitif, comme le dit sœur Rosa à Manuela. Cette dernière exprime alors son désir de créer une nouvelle famille dans un monde utopique, en dehors des autres. Sœur Rosa, à son tour, manifestera aussi le désir d'une nouvelle forme de famille, voire d'une maternité partagée :

Sour Rosa : Je vais l'appeler Esteban.

Manuela : Ton fils ? Et pourquoi donc ?

Sour Rosa : A cause du tien... Ce sera notre fils à toutes les deux.

Manuela s'attendrit. Elle lui frictionne le visage et les bras, manipulant son corps inerte.

Manuela : Ce serait bien !... Ah, si on pouvait être seules au monde, sans obligations, sans bonnes sœurs, ni famille, ni amis... Toi et ton enfant, pour moi toute seule !... Mais tu as une famille, Rosa... ${ }^{12}$

On l'aura compris, la famille traditionnelle apparaitrait comme la seule ombre au tableau idyllique dépeint par Manuela. Car dans les films d'Almodóvar, les personnages cherchent à constituer un noyau familial, en 
dehors de la famille traditionnelle, dont il est censé combler les failles. Les exemples des films d'Almodóvar trouvent un écho dans les mots de Judith Butler lorsqu'elle affirme que d'autres modes d'association intime que la famille traditionnelle constituent un "bouleversement" de la parenté traditionnelle qui déplace la supposition que les relations biologiques et sexuelles structurent la parenté de manière centrale ${ }^{13}{ }^{13}$ Ces nouvelles formes d'association pourraient être fondées ou non sur des « relations sexuelles durables ou exclusives »; elles remettent en cause la "place centrale du biologique et des relations sexuelles » dans la parenté, conférant ainsi "à la sexualité un domaine séparé de celui de la parenté », ce qui supposerait un élargissement du concept de famille. ${ }^{14}$ Et c'est précisément cet élargissement que propose Almodóvar.

11 "Qui que vous soyez, je me suis toujours fiée à la bonté des inconnus », dit Huma à deux reprises dans le film. ${ }^{15}$ Cette phrase de Tennessee Williams constitue en quelque sorte le leitmotiv du film et exprimerait cette quête d'une famille alternative, d'une famille d'adoption, sorte d'association par affinités électives. Cette phrase prend tout son sens la deuxième fois qu'Huma la prononce à l'intention de Manuela. Ce sens sera corroboré le lendemain lorsque Huma propose à Manuela de travailler avec elle en tant qu'assistante personnelle et que celle-ci lui répondra : "Tu ne me connais pas ». Comme Blanche dans la pièce, Huma confiera pleinement en Manuela : "Avec la démonstration d'hier soir, cela me suffit $"{ }^{16}$ Cette même phrase paraît sous-tendre la rencontre entre Manuela et sœur Rosa : si la jeune femme explique à sa mère que son travail est d'aider les gens, même si elle ne les connaît pas, c'est elle qui demandera de l'aide à Manuela alors qu'elles se connaissent à peine. Les attentes de Rosa envers Manuela dépassent de loin celles d'une simple connaissance, puisqu'elle lui demande, en somme, de s'impliquer dans sa vie, d'être sa mère de substitution. Si Manuela proteste devant la demande de sœur Rosa, elle finit par accepter ce rôle :
Ecoute, Rosa, tu es en train de me demander d'être ta mère. Tu n'as pas le droit ! Tu as déjà une mère, même si elle ne te plaît pas ! Les parents, on ne les choisit pas. On fait avec. (Tout à coup, Manuela serre Rosa dans ses bras et la supplie, au bord des larmes). Rosa, pour l'amour du ciel ! Ne me fais pas de chantage, s'il te plaît ! Elle continue à la serrer dans ses bras un moment. Rosa est toujours silencieuse. Les deux femmes se lâchent et commencent à marcher vers la sortie. Manuela a pris Rosa par la main. ${ }^{17}$

Malgré l'opposition initiale, on l'a vu, Manuela finira par assumer ce rôle, remplaçant avantageusement la famille biologique de Rosa. ${ }^{18}$ Comme le signale Vilma Navarro-Daniels, Manuela se crée une nouvelle famille, qui ne se résume pas à celle que nous venons de mentionner et qui inclurait -outre la transsexuelle Agrado- Huma Rojo, actrice lesbienne et alcoolique qui fera de Manuela sa femme de confiance, alors qu'elles se connaissent à peine. ${ }^{19}$

Mais la composante la plus subversive est la présence de personnages transgenres, transsexuels, de véritables personnages transgressifs qui se situent dans les marges des genres.

L'histoire de Manuela avec Lola est, à ce titre, très éclairante. Elle la raconte à sœur Rosa :

Sœur Rosa : Pourquoi tu as une dent contre Lola?

Manuela : Lola a ce qu'il y a de pire chez l'homme et ce qu'il y a de pire 
chez la femme !... Je vais te raconter une histoire. J'avais une amie dans mon village, en Argentine, qui s'est mariée très jeune. Au bout d'un an, son mari est parti travailler à Paris. Ils avaient convenu qu'il l'appellerait quand il aurait trouvé une situation. Deux ans passèrent. Le mari économisa un petit pécule et s'installa à Barcelone pour ouvrir un bar. Mon amie le rejoignit. Deux ans, ce n'est pas long, mais le mari avait changé...

Sœur Rosa : Et il ne l'aimait plus...

Manuela : Le changement était plutôt physique. Le mari s'était fait mettre des seins plus gros que ceux de sa femme.

Sœur Rosa : Ah... Je vois.

Manuela : Mon amie était très jeune, elle se retrouvait dans un pays étranger et elle ne connaissait personne. Mis à part les nouveaux seins, son mari n'avait pas tellement changé. Elle finit donc par l'accepter. Les femmes, on fait n'importe quoi pour ne pas être seules.

Sœur Rosa (ni surprise, ni scandalisée) : Nous, les femmes, nous sommes plus tolérantes, mais ça c'est une bonne chose.

Manuela : On est connes... et un peu gouines !... [...].20

On n'aura aucun mal à reconnaître dans l'histoire que Manuela raconte à sœur Rosa sa propre histoire. Manuela accepte son «mari-aux-nichons », elle fait avec. ${ }^{21}$ Cette histoire remonte à une vingtaine d'années auparavant. Manuela, en apprenant qu'elle est enceinte de Lola, fuit Barcelone pour s'installer à Madrid et offrir une vie meilleure à son fils. Cette même Lola, transsexuelle, prostituée et héroïnomane, qui n'a toujours pas changé de sexe biologique, sera le père du troisième Esteban de la lignée, né de Rosa. Ce n'est pas la première fois dans le cinéma d'Almodóvar qu'un personnage transgenre conçoit un enfant avec une femme, c'est le cas du travesti Letal dans Talons aiguilles, qui représente, comme le signale Smith, " la primauté du volontarisme, la liberté du sujet de se placer (lui ou elle) de n'importe quel côté de la frontière sexuelle $» .^{22}$ Par ailleurs, on peut s'interroger sur l'identité générique de personnages comme Lola et Agrado. Elles parlent d'elles au féminin mais ne sont pas allées jusqu'au bout dans leur démarche transsexuelle, ce qui fait d'elles des personnages transgressifs. Car si la transsexualité vise la normalité, qu'elle vise " à acquérir le genre que l'on a intérieurement et qui n'est pas marqué physiquement », ni Lola ni Agrado ne changent complètement de sexe. ${ }^{23}$ Par ailleurs, Agrado n'a aucune intention de se faire opérer, car les clients (elle se prostitue) les aiment « pneumatiques et bien pourvues ». ${ }^{24}$

Avec des personnages comme Lola, Agrado ou Letal, Almodóvar dépasse le clivage masculin/féminin, estompant les frontières entre sexe, genre et sexualité. ${ }^{25}$ Une scène très éclairante à ce titre est celle où la mère de Rosa surprend Manuela, le petit Esteban et la transsexuelle Lola, cet être étrange ${ }^{26}$ :

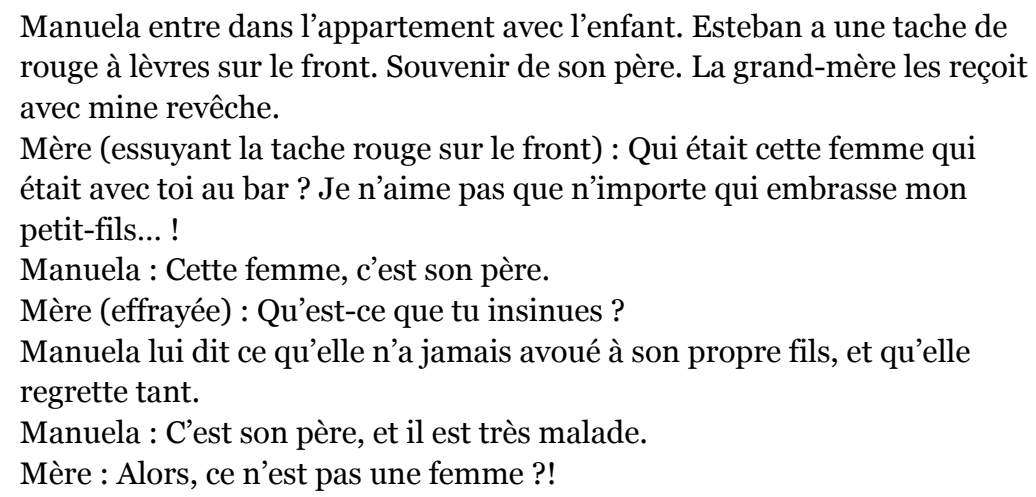


Manuela : Pas toujours.

La mère est horrifiée.

Mère : C'est ce monstre qui a tué ma fille !

Manuela se retire dans sa chambre, pour laisser la mère seule avec sa

douleur.

Manuela : n'y pensez plus, Rosa. ${ }^{27}$

Lola (comme Agrado) se place donc dans une zone d'indéfinition sexuelle d'autant plus floue que Lola devient mère et père en même temps : père puisque capable de féconder une jeune femme, et de surcroît bonne sœur de son état (par cela même Almodóvar dynamite tous les codes de la famille traditionnelle), mère de par son apparence physique. ${ }^{28}$ L'histoire se répète puisque sœur Rosa, comme Manuela, attend un enfant de Lola, qui continue visiblement d'avoir des rapports sexuels avec des femmes... tout en se prostituant, sûrement avec des hommes. Le réalisateur bouscule ainsi les codes : l'improbable couple de la bonne sœur et du transsexuel devient en quelque sorte un couple lesbien. Comme celui formé, avant Rosa, par Manuela et Lola. D'ailleurs, la réplique de Manuela, « on est [toutes] un peu gouines », expliquerait qu'elle, hétérosexuelle, s'accommode, du moins dans un premier temps, de cette relation. Les frontières de genre ne seraient pas si étanches que cela. ${ }^{29}$

Almodóvar est conscient du caractère transgressif du personnage de Lola et dépasse tous les clivages lorsqu'il donne à voir le désir de filiation comme un instinct presque animal, qui ne connait pas de genre :

[...] [L]e film parle par dessus tout de la mise au monde d'un être, de la maternité qui devient paternité et inversement. Je n'ose pas utiliser l'un ou l'autre de ces deux mots pour Lola. Le film dit aussi qu'au-delà des circonstances de la vie de chacun, il existe un instinct animal qui t'incite à engendrer et à défendre ce que tu as engendré, et à exercer des droits sur cet être. C'est ce que représente Lola et qui est peut-être ce qu'il y a de plus scandaleux dans le film, mais que je revendique de façon naturelle. Lola change sa nature, porte atteinte à son corps tout entier, mais il y a quelque chose en elle qui reste intact, et cela me touche, je ne saurais dire pourquoi. ${ }^{30}$

Dans Tout sur ma mère, le préfixe trans- est omniprésent, comme le rappelle Almodóvar lui-même lors d'un entretien avec Juan Cueto, journaliste d'El País ; préfixe que l'on retrouve dans des substantifs comme transsexuel, transplante (greffe) et transmission. ${ }^{31}$

En effet, Tout sur ma mère est une histoire de transmission. Transmission du virus du VIH, d'Esteban père au nouveau-né ; transmission du nom et du prénom paternel ; puis transmission du rôle maternel, puisqu'à la mort de Rosa et de Lola ce ne sont pas les parents biologiques qui prennent en charge le bébé, mais Manuela, étrangère au noyau familial (qui se résume à ses grandsparents maternels). C'est sa capacité à performer la maternité qui fera d'elle une mère. Une fois de plus, l'histoire se répète, car elle doit assumer cette seconde maternité toute seule, fuyant Barcelone pour amener le petit à Madrid, afin qu'il grandisse dans un environnement moins hostile. ${ }^{32}$

En définitive, les films d'Almodóvar dépassent le concept de famille traditionnelle et donnent à voir une autre modèle de famille, dans laquelle les liens du sang sont remplacés par d'autres liens affectifs, parfois d'amitié. Le réalisateur espagnol rompt également avec les stéréotypes de genre selon lesquels les femmes sont de bonnes mères ou destinées à l'être ou qu'elles 
auraient l'instinct maternel. Il introduit également des mères ou des parents transsexuels ou transgenres qui se montrent plus capables d'amour que beaucoup de familles traditionnelles. ${ }^{33}$ Comme le rappelle Pietsie Feenstra, les mères travesties ou transsexuelles du cinéma d'Almodóvar sont des « médiatrices », qui

\begin{abstract}
vivent en couple homosexuel, négocient l'impossibilité biologique de l'homme de porter des enfants, et expriment les sentiments maternels. Par ces corps almodovariens, des nouvelles figures mythiques sur la maternité sont créées, par ce médiateur qui se travestit en femme et se comporte comme une bonne mère. Un renvoi aux valeurs archétypales de la maternité, actualisées par les corps multisexuels ou la présence des couples homosexuels, impose une réflexion sur les attentes traditionnelles de la famille, sur la maternité, sur la paternité. ${ }^{34}$
\end{abstract}

Almodóvar dédie Tout sur ma mere « A Betty Davis, Gena Rowlands, Romy Schneider... A toutes ces actrices qui ont interprété des actrices, à toutes les femmes qui jouent, aux hommes qui jouent et se transforment en femme, à toutes les personnes qui veulent être mère. A ma mère $"{ }^{35}$ Cette dédicace met l'accent sur le caractère performatif du genre, sur la maternité comme un choix des personnes, quel que soit leur sexe, sur la vision « élargie » qu'il a de la famille. Maternité et filiation peuvent donc être des rôles, comme le genre.

Celui qui chantait aux temps de la Movida " Je vais être maman » sous le mode de la provocation, affirmera qu'il a toujours voulu être une mère célibataire. Mère, non pas père... les sentiments maternels ne correspondent pas toujours à l'identité générique.

Almodóvar donne un tour d'écrou aux traditionnels rôles génériques en offrant une vision queer du genre et de la filiation.

\title{
Notes
}

1 «S’il y a un élément clé dans mes films, c'est la maternité. La relation mère-fille est une relation compliquée par nature, alors que la relation entre grand-mère et petite-fille ne l'est pas. Il y a des mystères dans ce lien mère-fille, et c'est sans doute pour cela que j’y reviens. Je suis particulièrement ému, à la fin de Volver, quand Penélope et Carmen se serrent dans les bras l'une de l'autre. La fille dit alors à sa mère, dont elle a été séparée pendant longtemps : "Je ne sais pas comment j'ai pu vivre toutes ces années sans toi”. C'est une phrase très simple, mais elle me bouleverse. Elle parle de l'absence de la mère, qui a été si dure à vivre, et de la joie de pouvoir l'aimer » (http://www.telerama.fr/cinema/14920-

pedro_almodovar_et_carmen_maura_ai_une_vocation_de_mere_celibataire_pedro _almodovar.php).

2 Tina a changé de sexe par amour pour leur père, qui l'abandonnera par la suite. Sa vie sentimentale sera désormais une succession de déboires. Le spectateur l'apprend lorsqu'elle le raconte à son frère, devenu amnésique, pour essayer de l'aider à recouvrer la mémoire.

3 Judith Butler parle de la structure imitative du genre dans (entre autres) Trouble dans le genre, Paris, La Découverte / Poche, 2006, p. 256-266.

4 On voit très bien cela dans La mauvaise éducation, où Juan (qui se fait appeler Angel Andrade) assiste aux shows d'un travestit qui imite Sara Montiel afin de l'imiter et de copier ses mimiques pour les besoins du film d'Enrique Goded, dans lequel il campera le rôle d'un transsexuel.

5 « El artificio es la única verdad de Tina; el artificio, no la mentira: son dos cosas muy distintas. El artificio es su única verdad, y si el individuo no está loco, y el personaje que interpreta Carmen no lo está, se sabe artificial y goza con esa imitación de lo que es esencial en la mujer, de la parte más íntima femenina. A Carmen la obliga a imitar a una 
mujer, a gozar con esa imitación, a ser consciente de la parte kitsch que hay en esa imitación, renunciando siempre a la parodia, pero no al humor », [ "L'artifice est la seule vérité de Tina ; l'artifice, pas le mensonge. Ce sont des choses très distinctes. L'artifice est sa seule vérité, et si l'individu n'est pas fou, et le personnage qu'interprète Carmen ne l'est pas, il se sait artificiel et jouit de cette imitation de ce qui est essentiel chez la femme, de la partie la plus intime de la femme. Cela oblige Carmen à imiter une femme, à jouir de cette imitation, à être consciente de la part de kitsch qu'il y a dans cette imitation, renonçant toujours à la parodie mais non à l'humour ", traduit par nos soins]. Nuria Vidal, El cine de Pedro Almodóvar, Barcelona, Ed. Destino 1989, coll. Destinolibro, $\mathrm{n}^{\circ}$ 285, p. 213. Par ailleurs, Carmen Maura déclare avoir eu à l'esprit en tout moment qu'elle jouait le travelo ( « Te tienes que acordar todo el rato de que estás haciendo de travestón »), et que Pedro lui avait donné comme consigne de faire de la musculation et d'apprendre à frapper comme un homme. Ibid., p. 211 et 214.

6 Frédéric Strauss, Conversations avec Pedro Almodóvar, Ed. Cahiers du cinéma, 2000, nouvelle édition augmentée 2004, p. 72.

7 Ibid.

8 D'ailleurs, comme le signale Judith Butler, où est le vrai ? :« Le gai ou la lesbienne est donc à l'hétéroxexuel.le non pas ce que la copie est à l'original, mais plutôt ce que la copie est à la copie. La répétition parodique d'un original [...] », Judith Butler, Trouble dans le genre, op. cit., p. 107. Et elle va plus loin dans ce sens lorsqu'elle ajoute : «L'idée que je soutiens ici, à savoir que le genre est une parodie, ne présuppose pas l'existence d'un original qui serait imité par de telles identités parodiques. Au fond, la parodie porte sur l'idée même d'original ; tout comme la notion psychanalytique d'identification de genre renvoie au fantasme d'un fantasme -la transfiguration d'un Autre qui est toujours déjà une "figure" au double sens du terme-, la parodie du genre révèle que l'identité originale à partir de laquelle le genre se construit est une imitation sans original », ibid., p. 261.

9 Et j'applique ici le qualificatif et les commentaires de Nicolas Balutet dans son article " Performance du genre et métamorphoses du corps dans les Caraïbes ", in reCHERches Culture et Histoire dans l'Espace Roman, $\mathrm{n}^{\circ} 4$ "Les représentations du corps dans la littérature latino-américaine ", Université de Strasbourg, printemps 2010, p. 31.

10 Strauss, Ibid., p. 189.

11 Cf. Vilma Navarro-Daniels, "Tejiendo nuevas identidades: La red metaficcional e intertextual en Todo sobre mi madre de Pedro Almodóvar ", in http://www.lehman.cuny.edu/ciberletras/vo7/navarrodaniels.html.

12 Pedro Almodóvar, Tout sur ma mère, Scénario bilingue, Petite bibliothèque des Cahiers du cinéma, 2001.

13 Judith Butler, « Hors de soi », in Défaire le genre, Paris, Editions Amsterdam, 2012, p. 41.

14 Judith Butler, " La parenté est-elle toujours hétérosexuelle ? », in Défaire le genre, Ibid., p. 150.

15 C'est la phrase que le personnage de Blanche, joué par Huma Rojo, prononce dans la pièce Un Tramway nommé désir, à la représentation de laquelle assistent Manuela et son fils. Huma Rojo la prononce pour la $2^{\mathrm{e}}$ fois lors de sa rencontre avec Manuela. Sur le rôle de l'intertextualité, notamment de l'intertexte de Un Tramway nommé désir, de Tennessee Williams, dans Tout sur ma mère voir l'article de Vilma Navarro-Daniels, art. cit.

16 Almodóvar, Tout sur ma mère, Scénario bilingue, op. cit., p.105.

17 Ibid., p. 113-115.

18 Dans La loi du désir, Ada choisit, elle aussi, sa famille car elle préfère rester avec Tina, vu que ses grands-parents ne lui plaisent pas, comme le rappelle Tina.

19 « La pérdida de su hijo la lleva a Barcelona, entrando en contacto con el mundo de la prostitución, el SIDA y las drogas. Sin embargo, es en este entorno donde Manuela conforma su nueva familia, cuyos miembros son la Hermana Rosa - una monja embarazada y portadora del VIH-, la Agrado - un transexual que trabaja como prostituta- y Huma Rojo - una actriz lesbiana y alcohólica. Aunque esto por sí mismo transgrede el ideal tradicional de familia, la hipótesis que guía este estudio es que el particular uso que Almodóvar hace de la metaficción y de la intertextualidad es lo que le 
permite socavar profundamente ese convencionalismo ». [La perte de son fils l'amène à Barcelone, où elle entre en contact avec le monde de la prostitution, du SIDA et des drogues. Toutefois, c'est dans ce milieu que Manuela constituera sa nouvelle famille, dont les membres seront sœur Rosa - une nonne enceinte et porteuse du virus VIH-, Agrado - un transsexuel qui travaille en tant que prostitué- et Huma Rojo -actrice lesbienne et alcoolique. Bien que cela constitue en soi une transgression à l'idée traditionnelle de famille, l'hypothèse qui guide cette étude est que le particulier usage qu'Almodóvar fait de la métafiction et de l'intertextualité est ce qui permet de saper profondément ce conventionnalisme]. Vilma Navarro-Daniels, art. cit.

20 Almodóvar, scénario bilingue, op. cit., p. 107-109.

21 Ibid.

22 Smith, cité par Allison, in Mark Allison, Un laberinto español. Las películas de Pedro Almodóvar, Madrid, Ocho y medio, 2003, p. 123. C'est nous qui traduisons. Par ailleurs, comme le signale Allison, le personnage de Letal démontre ainsi que travestisme et homosexualité ne sont pas nécessairement liés. Allison, Ibid.

23 Nicolas Balutet, art. cit., p. 35.

24 Almodóvar, scénario bilingue, op. cit., p. 157.

$25 C f$. le mémoire de Denise Kolta, Les performances de l'identité : transtextualité et transgenre dans Talons aiguilles et Tout sur ma mère de Pedro Almodóvar, consultable à l'adresse suivante : http://www.archipel.uqam.ca/2717/1/M9377.pdf.Voir aussi Marie Piganiol, Transgenderism and Transsexuality in Almodovar's Movies, Amsterdam Social Science, Vol. 1 (2), 2009, p. 80.

26 Almodóvar, scénario bilingue, op. cit., p. 193.

27 Ibid.

28 Sur l'hybridation sexuelle de Lola et Agrado voir l'article de Pascale Thibaudeau " Greffes et transplantations de tissus filmiques : une métaphore à la manière de Pedro Almodóvar », Véronique Campan \& Gilles Ménégaldo (dir.), Du maniérisme au cinéma, La Licorne, Poitiers, MSHS, 2003, p. 195-211.

29 Almodóvar, scénario bilingue, op. cit., p. 107-109.

30 Strauss, op. cit., p. 159 et 162.

31 La greffe est l'un des fils conducteurs. Manuela est responsable des greffes dans son hôpital, anime des séminaires adressés aux docteurs et sera confrontée au don d'organes lorsque son fils décède.

La transmission occupe une place centrale dans le film, comme le signale Almodóvar : « [...] yo creo que aquí, en esta hora y pico, se trata exclusivamente del prefijo trans: transexualidad, transplantes, transmisión de sentimientos y virus, transtextualidad. En definitiva, el melodrama transgresor como transmodernidad » [je crois qu'ici, pendant cette heure et des poussières, il est exclusivement question du préfixe trans : transsexualité, greffes (transplantation), transmission de sentiments et de virus, transtextualité. En définitive, le mélodrame transgresseur en tant que trans-modernité], Juan Cueto, "Todo sobre mi madre. Perdonarlo todo ", El País, 4 décembre 2004, http://elpais.com/diario/2004/12/04/espectaculos/1102114805_850215.html. Au sujet des transsexuels et de la greffe, voir l'article de Pascale Thibaudeau " Greffes et transplantations de tissus filmiques ", in La Licorne, Du maniérisme au cinéma, Poitiers, MSHS, 2003.

32 « By the end of the film, Manuela is once more the mother of a son called Esteban, also fathered by Lola, but born to Rosa, a nun. However, there is no miraculous rebirth in which death is transcended or falsified. Rather, because of how Manuela acts, new life is found present in the reality of death and the presence she felt about her comes to be embodied once more. It may be that the materialization of the camera and the reembodiment of Esteban are both effects of a process whereby a certain kind of acting which finds its material in absence can sometimes create or realize its own reality. Examination of the various types of acting and role playing undertaken by the film's characters can help isolate the distinctive nature of such a reality-creating performance. All About My Mother suggest that women acting as mothers in this way give birth to and sustain life ». (Michael Sofair)

33 Pietsie Feenstra montre comment Almodóvar rompt avec l'archétype de la mère traditionnelle et du corps maternel : " L'archétype de la mère se base en général sur des 
valeurs protectrices envers l'enfant ; éduquer et nourrir sont considérés comme des gestes féminins. Bien que renvoyant à ces valeurs, les corps almodovariens ne donnent pas toujours une image traditionnelle. Dans La ley del deseo (1987), Carmen Maura représente par son corps un transsexuel et joue un personnage maternel ; elle est accompagnée de Bibi Andersen -une vraie transsexuelle- interprétant la mère biologique. Dans Todo sobre mi madre (1998), c'est le père qui se travestit en femme et une autre mère adopte son fils. Ces corps transsexuels et travestis expriment des sentiments maternels ; ce sont de bonnes mères, confirmant les valeurs archétypales, mais leurs corps s'opposent totalement à la normalité ; ainsi le corps interroge des valeurs traditionnelles par la pensée de son physique. Cette relation entre archétype et langage actuel, mise en image par le corps, illustre le processus de la construction des nouvelles figures mythiques, apportant un aspect historique au mythe ». Pietsie Feenstra, Les figures mythiques du cinéma espagnol (1975-1995). A corps perdus, Paris, L'Harmattan, 2006, p. 51.

34 Ibid., p. 54.

35 « A Betty Davis, Gena Rowlands, Romy Schneider... A todas las actrices que han hecho de actrices, a todas las mujeres que actúan, a los hombres que actúan y se convierten en mujeres, a todas las personas que quieren ser madres. A mi madre ». Générique de fin de Tout sur ma mère, (1999), DVD, 2000 Pathé.

\section{Pour citer cet article}

Référence électronique

Belén HERNANDEZ MARZAL, "Pedro Almodóvar ou la maternité performée ", Transtext(e)s Transcultures 跨文本跨文化 [En ligne], 8 | 2013, mis en ligne le 02 décembre 2013, consulté le 13 janvier 2014. URL : http://transtexts.revues.org/485

\section{Auteur}

\section{Belén HERNANDEZ MARZAL}

Belén Hernández Marzal est maître de conférences à l'Université Jean Moulin Lyon 3. Ses travaux de rechercher portent sur la poésie, les rapports cinéma-littérature et la métafiction. Elle est rattachée au groupe de recherche MARGE.

Belén Hernández Marzal is Maître de conférences at the University Jean Moulin Lyon 3. Her work focuses on poetry, on the relationships between literature and theatre, and metafiction. She is a member of MARGE (research center).

\section{Droits d'auteur}

(C) Tous droits réservés 